\title{
Review Article \\ Fractal-Based Methods and Inverse Problems for Differential Equations: Current State of the Art
}

\author{
Herb E. Kunze, ${ }^{1}$ Davide La Torre, ${ }^{2,3}$ Franklin Mendivil, ${ }^{4}$ \\ Manuel Ruiz Galán, ${ }^{5}$ and Rachad Zaki ${ }^{3}$ \\ ${ }^{1}$ Department of Mathematics and Statistics, University of Guelph, Guelph, ON, Canada N1G 2W1 \\ ${ }^{2}$ Department of Economics, Management, and Quantitative Methods, University of Milan, 20122 Milan, Italy \\ ${ }^{3}$ Department of Applied Mathematics and Sciences, Khalifa University, P.O. Box 127788, Abu Dhabi, UAE \\ ${ }^{4}$ Department of Mathematics and Statistics, Acadia University, Wolfville, NS, Canada B4P 2R6 \\ ${ }^{5}$ Department of Applied Mathematics, University of Granada, 18071 Granada, Spain
}

Correspondence should be addressed to Davide La Torre; davide.latorre@unimi.it

Received 18 March 2014; Accepted 30 July 2014; Published 20 November 2014

Academic Editor: Asier Ibeas

Copyright (C) 2014 Herb E. Kunze et al. This is an open access article distributed under the Creative Commons Attribution License, which permits unrestricted use, distribution, and reproduction in any medium, provided the original work is properly cited.

We illustrate, in this short survey, the current state of the art of fractal-based techniques and their application to the solution of inverse problems for ordinary and partial differential equations. We review several methods based on the Collage Theorem and its extensions. We also discuss two innovative applications: the first one is related to a vibrating string model while the second one considers a collage-based approach for solving inverse problems for partial differential equations on a perforated domain.

\section{Inverse Problems for Fixed Point Equations}

According to Keller [1], "we call two problems inverse of one another if the formulation of each involves all or part of the solution of the other. Often, for historical reasons, one of the two problems has been studied extensively for some time, while the other one is newer and not so well understood. In such cases, the former is called the direct problem, while the latter is the inverse problem." In practice, a general inverse problem asks us to use observed data to estimate parameters in the functional form of the governing model of the phenomenon under study [2-6].

There is a fundamental difference between the direct and the inverse problem; often the direct problem is wellposed while the corresponding inverse problem is ill-posed. Hadamard [7] introduced the concept of well-posed problem to describe a mathematical model that has the properties of existence, uniqueness, and stability of the solution. When one of these properties fails to hold, the mathematical model is said to be an ill-posed problem. There are several inverse problems in literature that are ill-posed whereas the corresponding direct problems are well-posed. The literature is rich in papers studying ad hoc methods to address ill-posed inverse problems by minimizing a suitable approximation error along with utilizing some regularization techniques [813].

Many inverse problems may be recast as the approximation of a target element $x$ in a complete metric space $(X, d)$ by the fixed point $\bar{x}$ of a contraction mapping $T: X \rightarrow$ $X$. Thanks to a simple consequence of Banach's Fixed Point Theorem known as the Collage Theorem, most practical methods of solving the inverse problem for fixed point equations seek an operator $T$ for which the collage distance $d(x, T x)$ is as small as possible.

Theorem 1 ("Collage Theorem" [14]). Let $(X, d)$ be a complete metric space and let $T: X \rightarrow X$ be a contraction mapping with contraction factor $c \in[0,1)$. Then, for any $x \in X$,

$$
d(x, \bar{x}) \leq \frac{1}{1-c} d(x, T x)
$$

where $\bar{x}$ is the fixed point of $T$. 
This vastly simplifies this type of inverse problem as it is much easier to estimate $d(x, T x)$ than it is to find the fixed point $\bar{x}$ and then compute $d(x, \bar{x})$. One now seeks a contraction mapping $T$ that minimizes the so-called collage error $d(x, T x)$, in other words, a mapping that sends the target $x$ as close as possible to itself. This is the essence of the method of collage coding which has been the basis of most, if not all, fractal image coding and compression methods. Barnsley et al. $[14,15]$ were the first to see the potential of using the Collage Theorem above for the purpose of fractal image approximation and fractal image coding [16]. However, this method of collage coding may be applied in other situations where contractive mappings are encountered.

We have shown this to be the case for inverse problems involving several families of differential equations and application to different areas: ordinary differential equations [17-19], Urison-type integral equations [20], random differential equations $[21,22]$, boundary value problems [23-25], parabolic partial differential equations [26], stochastic differential equations [27-29], and others [3,30]. In practical applications, from a family of contraction mappings $T_{\lambda}, \lambda \in \Lambda \subset$ $\mathbb{R}^{n}$, one wishes to find the parameter $\bar{\lambda}$ for which the approximation error $d\left(x, \bar{x}_{\lambda}\right)$ is as small as possible. In practice, the feasible set is often taken to be $\Lambda_{c}=\left\{\lambda \in \mathbb{R}^{n}: 0 \leq c_{\lambda} \leq\right.$ $c<1\}$ which guarantees the contractivity of $T_{\lambda}$ for any $\lambda \epsilon$ $\Lambda_{c}$. The main difference between this "collage" approach and the one based on the Tikhonov regularization is the following (see $[12,13])$ : in the collage approach, the constraint $\lambda \in \Lambda_{c}$ guarantees that $T_{\lambda}$ is a contraction and, therefore, it replaces the effect of the regularization term in the Tikhonov approach (see also $[6,8,11])$.

The collage-based inverse problem can be formulated as an optimization problem as follows:

$$
\min _{\lambda \in \Lambda_{c}} d\left(x, T_{\lambda} x\right)
$$

This is a nonlinear and nonsmooth optimization model. However, as the next sections below show, the above model (2) can often be reduced to a quadratic optimization program. Several algorithms can be used to solve it including, for instance, penalization methods and particle swarm ant colony techniques.

The paper is organized as follows. Section 2 presents the method for the case of differential equations while Section 3 illustrates the case of different families of partial differential equations (PDEs), namely, elliptic, parabolic, and hyperbolic equations. Section 4 presents applications to a randomly forced vibrating string and also to an inverse problem on perforated domains.

\section{Inverse Problems for IVPs by the Collage Theorem}

In [18], and subsequent works [17, 20-22, 25], the authors showed how collage coding could be used to solve inverse problems for systems of differential equations having the form

$$
\begin{aligned}
\dot{u} & =f(t, u), \\
u(0) & =u_{0},
\end{aligned}
$$

by reducing the problem to the corresponding Picard integral operator associated with it

$$
(T u)(t)=u_{0}+\int_{0}^{t} f(s, u(s)) d s .
$$

Let us recall the basic results in the case where $f$ belongs to $L^{2}$. Let us consider the complete metric space $C([0, T])$ endowed with the usual $d_{\infty}$ metric and assume that $f(t, x)$ is Lipschitz in the variable $x$; that is, there exists a $K \geq 0$ such that $\left|f\left(s, x_{1}\right)-f\left(s, x_{2}\right)\right| \leq K\left|x_{1}-x_{2}\right|$, for all $x_{1}, x_{2} \in \mathbb{R}$. For simplicity, we suppose that $x \in \mathbb{R}$, but the same consideration can be developed for the case of several variables. Under these hypotheses, $T$ is Lipschitz on the space $C([-\delta, \delta] \times[-M, M])$ for some $\delta$ and $M>0$.

Theorem 2 (see [18]). The function $T$ satisfies

$$
\|T u-T v\|_{2} \leq c\|u-v\|_{2}
$$

for all $u, v \in C([-\delta, \delta] \times[-M, M])$, where $c=\delta K$.

Now, let $\delta^{\prime}>0$ be such that $\delta^{\prime} K<1$. In order to solve the inverse problem for (4), we take the $L^{2}$ expansion of the function $f$. Let $\left\{\phi_{i}\right\}$ be a basis of functions in $L^{2}\left(\left[-\delta^{\prime}, \delta^{\prime}\right] \times\right.$ $[-M, M])$ and consider

$$
f_{\lambda}(s, x)=\sum_{i=1}^{+\infty} \lambda_{i} \phi_{i}(s, x) .
$$

Each sequence of coefficients $\lambda=\left\{\lambda_{i}\right\}_{i=1}^{+\infty}$ then defines a Picard operator $T_{\lambda}$. Suppose further that each function $\phi_{i}(s, x)$ is Lipschitz in $x$ with constants $K_{i}$.

Theorem 3 (see [18]). Let $K, \lambda \in \ell^{2}(\mathbb{R})$. Then,

$$
\left|f_{\lambda}\left(s, x_{1}\right)-f_{\lambda}\left(s, x_{2}\right)\right| \leq\|K\|_{2}\|\lambda\|_{2}\left|x_{1}-x_{2}\right|
$$

for all $s \in\left[-\delta^{\prime}, \delta^{\prime}\right]$ and $x_{1}, x_{2} \in[-M, M]$, where $\|K\|_{2}=$ $\left(\sum_{i=1}^{+\infty} K_{i}^{2}\right)^{1 / 2}$ and $\|\lambda\|_{2}=\left(\sum_{i=1}^{+\infty} \lambda_{i}^{2}\right)^{1 / 2}$.

Given a target solution $x$, we now seek to minimize the collage distance $\left\|u-T_{\lambda} u\right\|_{2}$. The square of the collage distance becomes

$$
\begin{aligned}
\Delta^{2}(\lambda) & =\left\|u-T_{\lambda} u\right\|_{2}^{2} \\
& =\int_{-\delta}^{\delta}\left|u(t)-\int_{0}^{t} \sum_{i=1}^{+\infty} \lambda_{i} \phi_{i}(s, u(s)) d s\right|^{2} d t
\end{aligned}
$$

and the inverse problem can be formulated as

$$
\min _{\lambda \in \Lambda} \Delta(\lambda)
$$


where $\Lambda=\left\{\lambda \in \ell^{2}(\mathbb{R}):\|\lambda\|_{2}\|K\|_{2}<1\right\}$. To solve this problem numerically, let us consider the first $n$ terms of the $L^{2}$ basis; in this case, the previous problem can be reduced to

$$
\min _{\lambda \in \widetilde{\Lambda}} \widetilde{\Delta}^{2}(\lambda)=\int_{-\delta}^{\delta}\left|x(t)-\int_{0}^{t} \sum_{i=1}^{n} \lambda_{i} \phi_{i}(s, x(s)) d s\right|^{2} d t,
$$

where $\tilde{\Lambda}=\left\{\lambda \in \mathbb{R}^{n}:\|\lambda\|_{2}\|K\|_{2}<1\right\}$. This is a classical quadratic optimization problem which can be solved by means of classical numerical methods. A penalized version of (10) is the following:

$$
\begin{aligned}
& \min _{\lambda \in \mathbb{R}^{n}} \widetilde{\Delta}^{2}(\lambda)+\sigma_{1} \max \left\{0,1-\|\lambda\|_{2}^{2}\|K\|_{2}^{2}\right\}^{2} \\
& +\sigma_{2} \sum_{i=1}^{n} \max \left\{0,-\lambda_{i}\right\}^{2} .
\end{aligned}
$$

Let $\widetilde{\Delta}_{\min }^{n}$ be the minimum value of $\widetilde{\Delta}$ over $\widetilde{\Lambda}$. This is a nonincreasing sequence of numbers (depending on $n$ ) and as shown in [16] it is possible to show that $\lim _{\inf }{ }_{n \rightarrow+\infty} \widetilde{\Delta}_{\min }^{n}=$ 0 . This means that the distance between the target element and the unknown solution of the differential equation can be made arbitrarily small. In Kunze et al. [21], the authors considered the case of inverse problems for random stochastic differential equations while in Capasso et al. [29] the case of stochastic differential equations is analyzed.

Example 4. Suppose that the stochastic process $X_{t}$ is believed to follow a geometric Brownian motion; then it satisfies the stochastic differential equation

$$
d X_{t}=a X_{t} d t+b X_{t} d W_{t}
$$

where $W_{t}$ is a Wiener process and the constants $a$ and $b$ are the percentage drift and the percentage volatility, respectively. We consider the following inverse problem: given realizations/paths $X_{t}^{i}, 1 \leq i \leq N$, estimate the values $a$ and $b$. Taking the expectation in (12), we see that $\mathbb{E}\left(X_{t}\right)$ satisfies the simple fixed point equation

$$
\mathbb{E}\left(X_{t}\right)=T\left(\mathbb{E}\left(X_{t}\right)\right)=X_{0}+\int_{0}^{t} a \mathbb{E}\left(X_{r}\right) d r .
$$

Hence, to solve the inverse problem, we construct the mean of the realizations

$$
X_{t}^{*}=\frac{1}{N} \sum_{i=1}^{N} X_{t}^{i}
$$

and use collage coding to determine the value of $a$ that minimizes the collage distance $d_{2}\left(X_{t}^{*}, T X_{t}^{*}\right)$. We can then estimate the value of $b$ by using the known formula $\operatorname{var}\left(X_{t}\right)=$ $e^{2 a t} X_{0}^{2}\left(e^{b^{2} t}-1\right)$, approximating $\operatorname{var}\left(X_{t}\right)$ from the realizations. As an example, we set $a=2, b=4$, and $X_{0}=1$ and then generate $N$ paths on $[0,1]$, dividing the interval into $M$ subintervals in order to simulate the Brownian motion on $[0,1]$. Beginning with these paths, we seek estimates of $a$ and $b$ using collage coding. Figure 1 shows five paths for the Brownian motion and the process $X_{t}$. Table 1 presents the numerical results of the example.

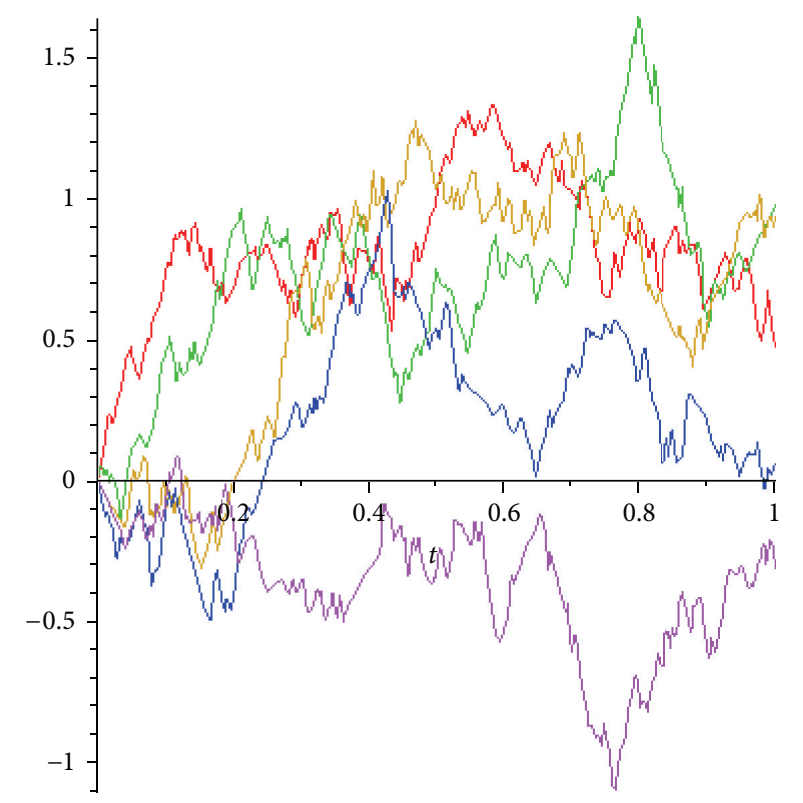

FIGURE 1: Different paths of the Brownian motion with $M=1000$ and $N=300$

TABLE 1: Minimal collage distance parameters for different $N$ and $M$, to five decimal places.

\begin{tabular}{lccc}
\hline$N$ & $M$ & $b$ & $a$ \\
\hline 100 & 300 & 3.78599 & 1.69618 \\
100 & 600 & 4.33750 & 1.78605 \\
100 & 1000 & 4.05374 & 1.85780 \\
300 & 300 & 3.34231 & 1.80282 \\
300 & 600 & 3.54219 & 1.81531 \\
300 & 1000 & 3.84973 & 1.78323 \\
\hline
\end{tabular}

\section{Inverse Problems for BVPs by the Generalized Collage Theorem}

3.1. Elliptic Equations. Let us consider the following variational equation:

$$
a(u, v)=\phi(v), \quad v \in H,
$$

where $\phi(v)$ and $a(u, v)$ are linear and bilinear maps, respectively, both defined on a Hilbert space $H$. Let us denote by $\langle\cdot, \cdot\rangle$ the inner product in $H,\|u\|^{2}=\langle u, u\rangle$ and $d(u, v)=\| u-$ $v \|$, for all $u, v \in H$. The inverse problem may now be viewed as follows: suppose that we have an observed solution $u$ and a given (restricted) family of bilinear functionals $a_{\lambda}(u, v)$, $\lambda \in \mathbb{R}^{n}$. We now seek "optimal" values of $\lambda$. The existence and uniqueness of solutions to this kind of equation are provided by the classical Lax-Milgram representation theorem. Suppose that we have a "target" element $u \in H$ and a family of bilinear functionals $a_{\lambda}$. Then, by the Lax-Milgram theorem, there exists a unique vector $u_{\lambda} \in H$ such that $\phi(v)=a_{\lambda}\left(u_{\lambda}, v\right)$ for all $v \in H$. We would like to determine if there exists a value of the parameter $\lambda$ such that $u_{\lambda}=u$ or, more realistically, such that $\left\|u_{\lambda}-u\right\|$ is small enough. The following theorem will be useful for the solution of this problem. 

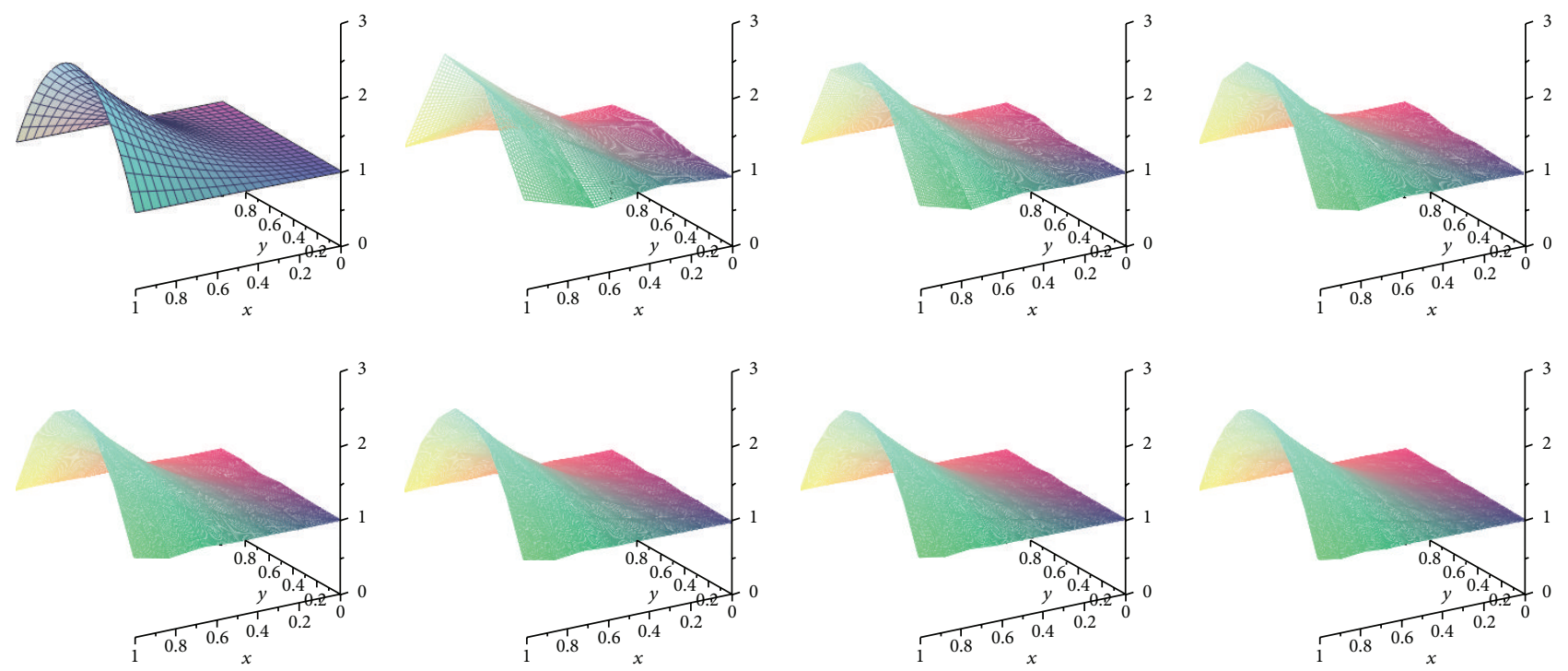

FIGURE 2: (Left to right, top to bottom) for two-dimensional Example 4, the graphs of our actual $\kappa(x, y)$, and the collage-coded approximations of $\kappa$ with $N=M=3$ through $N=M=9$.

Theorem 5 (Generalized Collage Theorem [24]). Suppose that $a_{\lambda}(u, v): \Lambda \times H \times H \rightarrow \mathbb{R}$ is a family of bilinear forms for all $\lambda \in \Lambda$ and $\phi: H \rightarrow \mathbb{R}$ is a given linear functional. Let $u_{\lambda}$ denote the solution of the equation $a_{\lambda}(u, v)=\phi(v)$ for all $v \in H$ as guaranteed by the Lax-Milgram theorem. Given a target element $u \in H$,

$$
\left\|u-u_{\lambda}\right\| \leq \frac{1}{m_{\lambda}} F(\lambda)
$$

where

$$
F(\lambda)=\sup _{v \in H,\|v\|=1}\left|a_{\lambda}(u, v)-\phi(v)\right| .
$$

In order to ensure that the approximation $u_{\lambda}$ is close to a target element $u \in H$, we can, by the Generalized Collage Theorem, try to make the term $F(\lambda) / m_{\lambda}$ as close to zero as possible. The appearance of the $m_{\lambda}$ factor complicates the procedure as does the factor $1 /(1-c)$ in standard collage coding, that is, (1). If inf ${ }_{\lambda \in \Lambda} m_{\lambda} \geq m>0$, then the inverse problem can be reduced to the minimization of the function $F(\lambda)$ on the space $\Lambda$; that is,

$$
\min _{\lambda \in \Lambda} F(\lambda) .
$$

The choice of $\lambda$ according to (18) for minimizing the residual is, in general, not stabilizing (see [8]). However, as the next sections show, under the condition $\inf _{\lambda \in \Lambda} m_{\lambda} \geq m>0$, our approach is stable. Following our earlier studies of inverse problems using fixed points of contraction mappings, we will refer to the minimization of the functional $F(\lambda)$ as a "generalized collage method." Such an optimization problem has a solution that can be approximated with a suitable discrete and quadratic program, derived from the application of the Generalized Collage Theorem and an adequate use of an orthonormal basis in the Hilbert space $H$, as seen in [24].
Example 6. We now present an inverse problem for the twodimensional steady-state diffusion equation. With $D=\{0<$ $x, y<1\}$,

$$
\begin{gathered}
-\nabla \cdot(\kappa(x, y) \nabla u(x, y))+q(x, y) u(x, y)=f(x, y), \\
(x, y) \in D, \\
u(x, y)=0, \quad(x, y) \in \partial D,
\end{gathered}
$$

where $\kappa(x, y)$ and $q(x, y)$ vary in both $x$ and $y$. Given $u(x, y), q(x, y)$, and $f(x, y)$ on $[0,1]^{2}$, we wish to find an approximation of $\kappa(x, y)$. As the first example, we set $u(x, y)=\sin (\pi x) \sin (\pi y), q(x, y)=0$, and use the function $\kappa(x, y)=1+6 x^{2} y(1-y)$ to determine $f(x, y)$ via (29). Now, given the functions $u(x, y), f(x, y)$, and $q(x, y)$, we seek to approximate $\kappa(x, y)$. This inverse problem is treated as Example 3 in [9], using a modified Uzawa algorithm. In Figure 2, we present graphs of our actual $\kappa(x, y)$, as well as the results obtained by minimizing (18). For $N$ and $M$ fixed natural numbers, we define $h_{x}=1 / N$ and $h_{y}=1 / M$, as well as the $(N+1)(M+1)$ nodes in $[0,1]^{2}$ as follows:

$$
\left(x_{i}, y_{j}\right)=\left(i h_{x}, j h_{y}\right), \quad 0 \leq i \leq N, 0 \leq j \leq M .
$$

The corresponding finite element basis functions $\xi_{i j}(x, y)$ are pyramids with hexagonal bases, such that $\xi_{i j}\left(x_{i}, y_{j}\right)=1$ and $\xi_{i j}\left(x_{k}, y_{l}\right)=0$ for $k \neq i, l \neq j$ (see [24] for more details on this).

Next, we perturb the target function $u(x, y)$, leaving $f(x, y)$ and $q(x, y)$ exact. Table 2 presents the $L^{2}$ error $\| u-$ $u_{\text {noisy }} \|$ between the true solution $u$ and the noised target $u_{\text {noisy }}$ and the resulting error $\left\|\kappa-\kappa_{\text {collage }}\right\|$ between the true $\kappa$ and the collage-coded approximation $\kappa_{\text {collage }}$ for numerous cases of $N$ and $M$. Note that $\|\kappa\|_{2}=1.38082$ and $\|u\|_{2}=0.5$. 
TABLE 2: Numerical results for the inverse problem with different levels of noise.

\begin{tabular}{lccc}
\hline$N=M$ & & $\left\|\kappa-\kappa_{\text {collage }}\right\|_{2}$ & $\left\|u-u_{\text {noisy }}\right\|_{2}=0.05$ \\
\hline 3 & $u_{\text {noisy }}=u$ & $\left\|u-u_{\text {noisy }}\right\|_{2}=0.025$ & 0.17050 \\
4 & 0.06306 & 0.09993 & 0.15561 \\
5 & 0.03480 & 0.07924 & 0.15128 \\
6 & 0.02246 & 0.07275 & 0.15065 \\
7 & 0.01564 & 0.07118 & 0.15039 \\
8 & 0.01160 & 0.07051 & 0.15014 \\
9 & 0.00902 & 0.07008 & 0.14996 \\
\hline
\end{tabular}
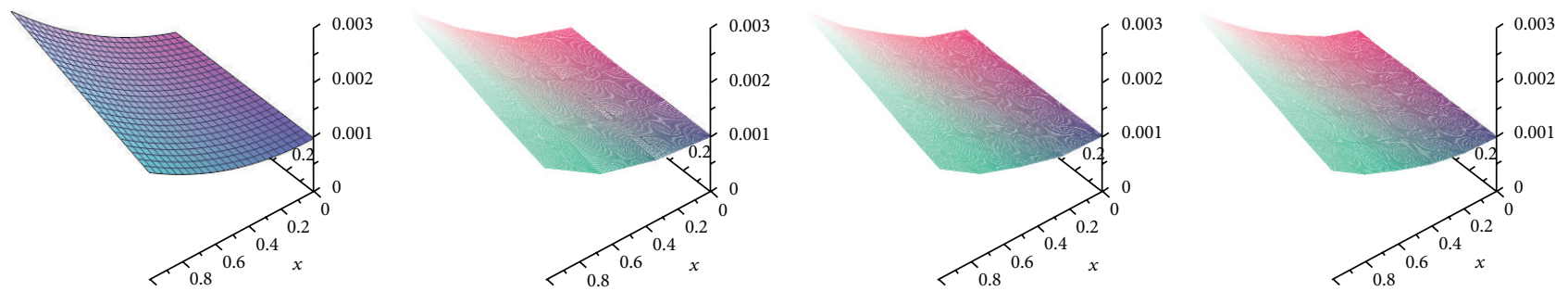

FIGURE 3: (Left to right) for two-dimensional Example 6, the graphs of our actual $\kappa(x, y)$, and the collage-coded approximations of $\kappa$ with $N=M=3,4,5$.

As the second example, let us follow Example 7 of [10], and we set $u(x, y)=\sin (\pi x) \sin (\pi y), q(x, y)=4+\cos (\pi x y)$, and $\kappa(x, y)=\left(1+x^{2}+x y\right) / 1000$. With these choices, we determine the function $f(x, y)$. The inverse problem is to estimate $\kappa(x, y)$ when given $u(x, y), f(x, y)$, and $q(x, y)$. In Figure 3, we present graphs of the results obtained by minimizing (18) with $N=M=3$ through $N=M=5$.

These results have been extended to a wider class of elliptic equations problems in [31,32], by considering not only Hilbert but also reflexive Banach spaces, and even replacing the primal variational formulation of such a problem, (15), with a more general constrained variational one. Let us mention that this kind of formulation arises, for instance, when the boundary constraints are weakly imposed.

Theorem 7 (see $[32,33])$. Let $X, Y, Z$, and $W$ be reflexive Banach spaces, let $\phi: Y \rightarrow \mathbb{R}$ and $\psi: W \rightarrow \mathbb{R}$ be bounded and linear functionals, and let $\Lambda$ be a nonempty set and assume that for each $\lambda \in \Lambda, \alpha_{\lambda}$ and $\beta_{\lambda}$ are positive real numbers and $a_{\lambda}: X \times Y \rightarrow \mathbb{R}, b_{\lambda}: Y \times Z \rightarrow \mathbb{R}$, and $c_{\lambda}: X \times W \rightarrow \mathbb{R}$ are bounded and bilinear forms. If one writes

$$
\begin{aligned}
& K_{b_{\lambda}}:=\left\{y \in Y: b_{\lambda}(y, z)=0, \forall z \in Z\right\}, \\
& K_{c_{\lambda}}:=\left\{x \in X: c_{j}(x, w)=0, \forall w \in W\right\}
\end{aligned}
$$

and suppose that

$$
\begin{aligned}
& x \in K_{c_{\lambda}}, \quad a_{\lambda}(x, y)=0, \quad \forall y \in K_{b_{\lambda}} \Longrightarrow x=0, \\
& y \in K_{b_{\lambda}} \Longrightarrow \alpha_{\lambda}\|y\| \leq \sup _{x \in K_{c^{\prime}},\|x\|=1}\left\|a_{\lambda}(x, y)\right\|,
\end{aligned}
$$

$$
w \in W \Longrightarrow \beta_{\lambda}\|w\| \leq \sup _{x \in X,\|x\|=1}\left\|c_{\lambda}(x, w)\right\|
$$

then the constrained variational equation

$$
x \in X \text { with }\left\{\begin{array}{l}
y \in K_{b_{\lambda}} \Longrightarrow a_{\lambda}(x, y)=\phi(y) \\
w \in W \Longrightarrow c_{\lambda}(x, w)=\psi(w)
\end{array}\right.
$$

admits a unique solution $x_{\lambda} \in X$ and in addition for all $x_{0} \in X$ there holds

$$
\begin{aligned}
\left\|x_{\lambda}-x_{0}\right\| \leq & \frac{1}{\alpha_{\lambda}} \sup _{y \in Y,\|y\|=1}\left|\phi(y)-a_{\lambda}\left(x_{0}, y\right)\right| \\
& +\frac{1}{\beta_{\lambda}}\left(1+\frac{\left\|a_{\lambda}\right\|}{\alpha_{\lambda}}\right) \sup _{w \in W,\|w\|=1}\left|\psi(w)-c_{\lambda}\left(x_{0}, w\right)\right| .
\end{aligned}
$$

If $\inf _{\lambda \in \Lambda} \alpha_{\lambda}>0, \inf _{\lambda \in \Lambda} \beta_{\lambda}>0$, and $\sup _{\lambda \in \Lambda}\left\|a_{\lambda}\right\|<\infty$, then the corresponding inverse problem leads to the minimization problem

$$
\begin{aligned}
\min _{\lambda \in \Lambda}\left(\sup _{y \in Y,\|y\|=1}\left|\phi(y)-a_{\lambda}\left(x_{0}, y\right)\right|\right. & \\
& \left.+\sup _{w \in W,\|w\|=1}\left|\psi(w)-c_{\lambda}\left(x_{0}, w\right)\right|\right),
\end{aligned}
$$

for which an approximated quadratic program follows from the preceding collage-type result and some properties of Schauder bases in the involved reflexive Banach spaces (see [32] for the details). 
3.2. Parabolic Equations. Suppose that we have a given Hilbert space $H$ and let us consider the following abstract formulation of a parabolic equation:

$$
\begin{aligned}
\left\langle\frac{d}{d t} u, v\right\rangle & =\psi(v)+a(u, v), \\
u(0) & =f
\end{aligned}
$$

where $\psi: H \rightarrow \mathbb{R}$ is a linear functional, $a: H \times H \rightarrow \mathbb{R}$ is a bilinear form, and $f \in H$ is an initial condition. The aim of the inverse problem for the above system of (29) consists of getting an approximation of the coefficients and parameters starting from a sample of observations of a target $u \in H$. To do this, let us consider a family of bilinear functionals $a_{\lambda}$ and let $u_{\lambda}$ be the solution to

$$
\begin{aligned}
\left\langle\frac{d}{d t} u_{\lambda}, v\right\rangle & =\psi(v)+a_{\lambda}\left(u_{\lambda}, v\right) \\
u_{0} & =f .
\end{aligned}
$$

We would like to determine if there exists a value of the parameter $\lambda$ such that $u_{\lambda}=u$ or, more realistically, such that $\left\|u_{\lambda}-u\right\|$ is small enough. To this end, Theorem 8 states that the distance between the target solution $u$ and the solution $u_{\lambda}$ of (27) can be reduced by minimizing a functional which depends on parameters.

Theorem 8. Let $u:[0, T] \rightarrow L^{2}(D)$ be the target solution which satisfies the initial condition in (29) and suppose that $(d / d t) u$ exists and belongs to $H$. Suppose that $a_{\lambda}(u, v): \Lambda \times$ $H \times H \rightarrow \mathbb{R}$ is a family of bilinear forms for all $\lambda \in \Lambda$. One has the following result:

$$
\begin{aligned}
\int_{0}^{T}\left\|u-u_{\lambda}\right\|_{H} d t \leq \frac{1}{m_{\lambda}^{2}} \int_{0}^{T} & \left(\sup _{\|v\|=1}\left\langle\frac{d}{d t} u, v\right\rangle\right. \\
& \left.-\psi(v)-a_{\lambda}(u, v)\right)^{2} d t
\end{aligned}
$$

where $u_{\lambda}$ is the solution of $(27)$ s.t. $u_{\lambda}(0)=u(0)$ and $u_{\lambda}(T)=$ $u(T)$.

Example 9. Let us consider the following equation:

$$
\begin{aligned}
& u_{t}=\left(k(x) u_{x}\right)_{x}+g(x, t), \quad 0<x<1, \\
& u_{0}=0, \\
& u_{1}=0
\end{aligned}
$$

where $g(x, t)=t x(1-x)$, subject to $u(x, 0)=10 \sin (\pi x)$ and $u(0, t)=u(1, t)=0$. We set $k(x)=1+3 x+2 x^{2}$, solve the problem for $u(x, t)$, and sample the solution at $N^{2}$ uniformly positioned grid points for $(x, t) \in[0,1]^{2}$ to generate a collection of targets. Given this data and $g(x, t)$, we then seek an estimation of $k(x)$ in the form $k(x)=k_{0}+k_{1} x+k_{2} x^{2}$. The results we obtain through the generalized collage method are summarized in Table 3. As for the elliptic case, the table shows that the method subject to noisy perturbations is stable.
TABLE 3: Collage coding results for the parabolic equation in Example 9.

\begin{tabular}{lcccc}
\hline Noise $\varepsilon$ & $N$ & $k_{0}$ & $k_{1}$ & $k_{2}$ \\
\hline 0 & 10 & 0.87168 & 2.90700 & 0.21353 \\
0 & 20 & 0.93457 & 2.97239 & 1.49201 \\
0 & 30 & 0.94479 & 2.98304 & 1.76421 \\
0 & 40 & 0.94347 & 2.97346 & 1.85572 \\
0.01 & 10 & 0.87573 & 2.82810 & 0.33923 \\
0.01 & 20 & 0.92931 & 2.91536 & 1.32864 \\
0.01 & 30 & 0.92895 & 2.84553 & 0.59199 \\
0.10 & 10 & 0.90537 & 1.97162 & 0.59043 \\
0.10 & 20 & 0.77752 & 0.92051 & -0.77746 \\
0.10 & 30 & 0.60504 & -0.12677 & -0.14565 \\
\hline
\end{tabular}

3.3. Hyperbolic Equations. Let us now consider the following weakly formulated hyperbolic equation:

$$
\begin{aligned}
\left\langle\frac{d^{2}}{d t^{2}} u, v\right\rangle & =\psi(v)+a(u, v), \\
u(0) & =f, \\
\frac{d}{d t} u(0) & =g,
\end{aligned}
$$

where $\psi: H \rightarrow \mathbb{R}$ is a linear functional, $a: H \times H \rightarrow \mathbb{R}$ is a bilinear form, and $f, g \in H$ define the initial conditions. As in the previous sections, the aim of the inverse problem for the above system of equations consists of reconstructing the coefficients starting from a sample of observations of a target $u \in H$. We consider a family of bilinear functionals $a_{\lambda}$ and let $u_{\lambda}$ be the solution to

$$
\begin{aligned}
\left\langle\frac{d^{2}}{d t^{2}} u_{\lambda}, v\right\rangle & =\psi(v)+a_{\lambda}\left(u_{\lambda}, v\right), \\
u_{0} & =f, \\
\frac{d}{d t} u(0) & =g .
\end{aligned}
$$

We would like to determine if there exists a value of the parameter $\lambda$ such that $u_{\lambda}=u$ or, more realistically, such that $\left\|u_{\lambda}-u\right\|$ is small enough. Theorem 10 states that the distance between the target solution $u$ and the solution $u_{\lambda}$ of (31) can be reduced by minimizing a functional which depends on parameters.

Theorem 10. Let $u:[0, T] \rightarrow L^{2}(D)$ be the target solution which satisfies the initial condition in (30) and suppose that $\left(d^{2} / d t^{2}\right) u$ exists and belongs to $H$. Suppose that there exists a family of $m_{\lambda}>0$ such that $a_{\lambda}(v, v) \geq m_{\lambda}\|v\|^{2}$ for all $v \in H$. One has the following result:

$$
\begin{aligned}
& \int_{0}^{T}\left\|u-u_{\lambda}\right\|^{2} d t \\
& \quad \leq \frac{1}{m_{\lambda}^{2}} \int_{0}^{T}\left(\sup _{\|v\|=1}\left\langle\frac{d^{2}}{d t^{2}} u, v\right\rangle-\psi(v)-a(u, v)\right)^{2} d t
\end{aligned}
$$


TABLE 4: Collage coding results for the hyperbolic equation in Example 11.

\begin{tabular}{lcccc}
\hline Noise $\varepsilon$ & $N$ & $k_{0}$ & $k_{1}$ & $k_{2}$ \\
\hline 0 & 10 & 0.87168 & 2.90700 & 0.21353 \\
0 & 20 & 0.93457 & 2.97239 & 1.49201 \\
0 & 30 & 0.94479 & 2.98304 & 1.76421 \\
0 & 40 & 0.94347 & 2.97346 & 1.85572 \\
0.01 & 10 & 0.87573 & 2.82810 & 0.33923 \\
0.01 & 20 & 0.92931 & 2.91536 & 1.32864 \\
0.01 & 30 & 0.92895 & 2.84553 & 0.59199 \\
0.10 & 10 & 0.90537 & 1.97162 & 0.59043 \\
0.10 & 20 & 0.77752 & 0.92051 & -0.77746 \\
0.10 & 30 & 0.60504 & -0.12677 & -0.14565 \\
\hline
\end{tabular}

where $u_{\lambda}$ is the solution of $(31)$ s.t. $u(0)=u_{\lambda}(0)$ and $u(T)=$ $u_{\lambda}(T)$.

Example 11. We adjust Example 9, considering

$$
u_{t t}-\left(k(x) u_{x}\right)_{x}=g(x, t),
$$

where $g(x, t)=t x(1-x)$, subject to $u(x, 0)=\sin (\pi x)$ and $u_{t}(x, 0)=0$ and $u(0, t)=u(1, t)=0$. We set $k(x)=1+3 x+$ $2 x^{2}$ and construct target data as in Example 9 and then seek to recover $k(x)=k_{0}+k_{1} x+k_{2} x^{2}$ given this data and $g(x, t)$. The results we get from the generalized collage method are summarized in Table 4, which again proves the stability of the method under noise.

\section{Application}

4.1. The Vibrating String Driven by a Stochastic Process. Before stating and solving two inverse problems, we begin by giving the details and motivations for the specific model we are interested in studying. We consider the following system of coupled differential equations. The first one is a stochastic differential equation and the second one is a hyperbolic partial differential equation. On a domain $D \subset \mathbb{R}^{d}$, we have the equations

$$
\begin{gathered}
d X_{t}=\left[\int_{D} g(u(t, y)) d \mu_{t}(y)\right] X_{t} d t+X_{t} d B_{t}, \\
X_{t=0}=X_{0}, \\
\frac{d^{2}}{d t^{2}} u(t, y)+\nabla_{y}\left(\kappa_{1}(y) \nabla_{y} u(t, y)\right)=\kappa_{2}(y) \delta_{X_{t}}(y), \\
u(0, y)=\phi_{1}(y), \\
\frac{\partial u}{\partial n}(t, y)=\phi_{2}(t, y), \quad(t, y) \in[0, T] \times \partial D,
\end{gathered}
$$

where $\mu_{t}$ is the law of $X_{t}$ and $\delta_{X_{t}}$ is the Dirac delta "function" at the point $X_{t}$.
For instance, imagine we have a flexible string directed along the $x$-axis, with the string kept stretched by a constant horizontal tension, and forced to vibrate perpendicularly to the $x$-axis under random force $F(x, t)$. If $u$ denotes the displacement of a point $x$ at time $t$, it is well known that $u$ satisfies the following equation:

$$
\frac{\partial^{2}}{\partial t^{2}} u-\frac{\partial^{2}}{\partial x^{2}} u=F(x, t),
$$

where $x \in D$ and $t>0$. In our model, we suppose that $F(y, t)=\kappa_{2}(y) \delta_{X_{t}}(y)$, where $X_{t}$ is a stochastic process which is a solution of the stochastic differential equation (34). In other words, the hyperbolic equation has a forcing term that is driven by this stochastic process. The random vibration on an infinite string has received recent attention (see [34, 35]). Figure 4 presents some snapshots of the displacement for the related finite string problem.

In the next sections, we present a solution method for solving two different parameter identification problems for this system of coupled differential equations: one for $\kappa_{1}$ and one for $g$. Both of these two methods are based on the numerical schemes which have been presented in the previous sections.

Before we begin the analysis, a few words about (35) are in order, since this equation contains the generalized function $\delta_{X_{t}}$. That is, $\delta_{X_{t}}(y)$ has a meaning only when it is integrated with respect to a test function $\theta(y)$. Thus, the meaning of (35) is that for each $\theta \in H^{1}(D)$ we have

$$
\begin{array}{r}
\int_{D} \theta(y)\left(\frac{d^{2}}{d t^{2}} u(t, y)+\nabla_{y}\left(\kappa_{1}(y) \nabla_{y} u(t, y)\right)\right) d y \\
=\int_{D} \theta(y) \kappa_{2}(y) \delta_{X_{t}}(y) d y=\theta\left(X_{t}\right) \kappa_{2}\left(X_{t}\right) .
\end{array}
$$

4.1.1. A Parameter Identification Model for $\kappa_{1}$. For this parameter identification problem, we seek to estimate $\kappa_{1}$ given $\kappa_{2}$, $g$, and the observations of $X_{t}$. From this data, we recover the density $f_{X_{t}}$ of the process $X_{t}$. Averaging equation (35) allows one to get a simpler model for the parameter identification problem (see [36]). This replaces the quantity $\delta_{X_{t}}$ with its expectation $\mathbb{E}\left(\delta_{X_{t}}(y)\right)$. Since $X_{t}$ is absolutely continuous, then $\mathbb{E}\left(\delta_{X_{t}}(y)\right)=f_{X_{t}}(y)$, the density of the distribution of $X_{t}$. The previous model can therefore be rewritten in an averaged form as

$$
\begin{aligned}
& d X_{t}=\left[\int_{D} g(\tilde{u}(t, y)) f_{X_{t}}(y) d y\right] X_{t} d t+X_{t} d B_{t}, \\
& X_{t=0}=X_{0},
\end{aligned}
$$

coupled with the deterministic PDE

$$
\begin{gathered}
\frac{d^{2}}{d t^{2}} \tilde{u}(t, y)+\nabla\left(\kappa_{1}(y) \nabla_{y} \tilde{u}(t, y)\right)=\kappa_{2}(y) f_{X_{t}}(y), \\
(t, y) \in[0, T] \times D \\
\tilde{u}(0, y)=\phi_{1}(y),
\end{gathered}
$$



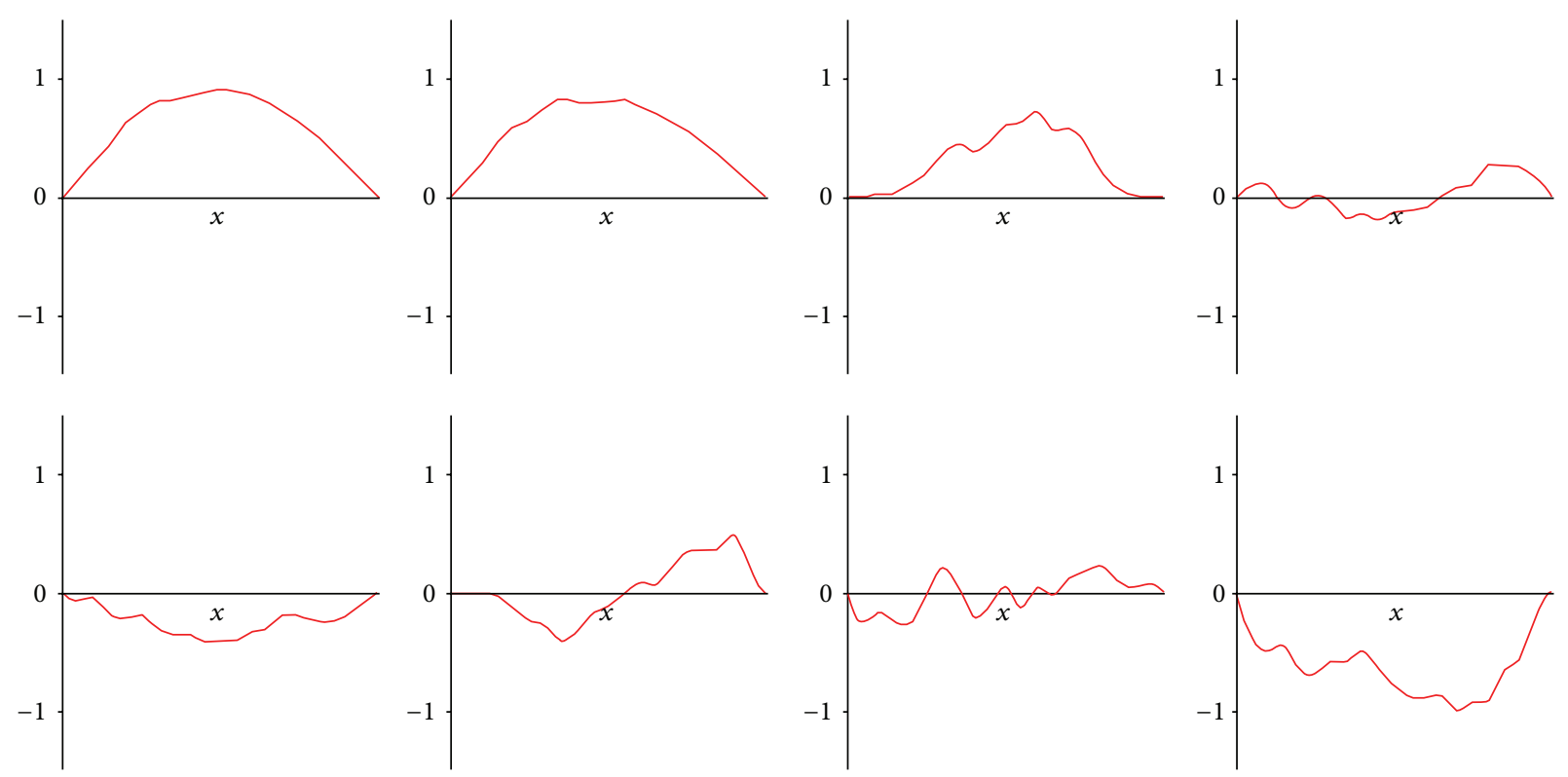

FIGURE 4: Snapshots of a randomly forced vibrating string, with time increasing from left to right and top to bottom.

Note that the averaged equation, (39), has solutions in the usual sense. For this particular case, we have $\mathbb{E}(u(t, y))=$ $\widetilde{u}(t, y)$. To see this, just notice that the operator $d^{2} / d t^{2}-\Delta_{y}$ is linear, so

$$
\begin{aligned}
& \mathbb{E}\left(\frac{d^{2}}{d t^{2}} u+\nabla_{y}\left(\kappa_{1}(y) \nabla_{y} u\right)\right) \\
& =\frac{d^{2}}{d t^{2}} \mathbb{E}(u)+\nabla_{y}\left(\kappa_{1}(y) \nabla_{y} \mathbb{E} u\right) .
\end{aligned}
$$

Furthermore,

$$
\mathbb{E}\left(\int_{D} \theta(y) \kappa_{2}(y) \delta_{X_{t}}(y) d y\right)=\int_{D} \theta(y) \kappa_{2}(y) f_{X_{t}}(y) d y,
$$

since $\kappa_{1}$ and $\kappa_{2}$ are deterministic functions. Thus, $\mathbb{E}(u)$ is the solution to the deterministic PDE

$$
\begin{array}{r}
\frac{d^{2}}{d t^{2}} \mathbb{E}(u(t, y))-\kappa_{1}(y) \Delta_{y} \mathbb{E}(u(t, y))=\kappa_{2}(y) f_{X_{t}}(y), \\
(t, y) \in[0, T] \times D, \\
\mathbb{E}(u(0, y))=\phi_{1}(y), \\
\frac{\partial \mathbb{E}(u)}{\partial n}(t, y)=\phi_{2}(t, y), \quad(t, y) \in[0, T] \times \partial D .
\end{array}
$$

However, this is clearly the same PDE for which $\widetilde{u}$ is the solution. Thus, we must have $\mathbb{E}(u)=\widetilde{u}$. This inverse problem can be solved using the techniques illustrated in a previous section on hyperbolic differential equations.

4.1.2. A Parameter Identification Problem for $g$. For this parameter identification problem, we assume that $\kappa_{1}$ and $\kappa_{2}$ are known. The aim of the inverse problem consists of recovering the functional form of $g$ and the density $f_{X_{t}}$ of the process $X_{t}$ starting from a sample of observations of the random process $u(t, y, \omega)$, say $\left(u\left(t, y, \omega_{1}\right), \ldots, u\left(t, y, \omega_{n}\right)\right)$. We do this in several steps. Let us denote by $\widetilde{u}=\mathbb{E}(u(t, y, \cdot))$. The first step is to use $u(t, y)$ and (35) to obtain an estimate of the distribution of $X_{t}$. Let us consider the averaged equation

$$
\begin{aligned}
d X_{t} & =\left[\int_{D} g(\tilde{u}(t, y)) f_{X_{t}}(y) d y\right] X_{t} d t+X_{t} d B_{t}, \\
X_{t=0} & =X_{0},
\end{aligned}
$$

coupled with the deterministic PDE

$$
\begin{array}{r}
\frac{d^{2}}{d t^{2}} \widetilde{u}(t, y)-\kappa_{1}(y) \Delta_{y} \widetilde{u}(t, y)=\kappa_{2}(y) f_{X_{t}}(y), \\
(t, y) \in[0, T] \times D, \\
\widetilde{u}(0, y)=\phi_{1}(y), \\
\frac{\partial \widetilde{u}}{\partial n}(t, y)=\phi_{2}(t, y), \quad(t, y) \in[0, T] \times \partial D .
\end{array}
$$

Dividing the left side of the PDF in (44) by $\kappa_{2}$, we get $f_{X_{t}}$. Let us go back to the stochastic differential equation (43), and let us take the expectation of both sides; we get

$$
\begin{aligned}
& d \mathbb{E}\left(X_{t}\right)=\left[\int_{D} g(\widetilde{u}(t, y)) f_{X_{t}}(y) d y\right] \mathbb{E}\left(X_{t}\right) d t, \\
& \mathbb{E}\left(X_{t=0}\right)=\mathbb{E}\left(X_{0}\right) .
\end{aligned}
$$

We remember that the following relationship holds between the expectation of a random variable and its density:

$$
\mathbb{E}\left(X_{t}\right)=\int_{\mathbb{R}^{d}} y f_{X_{t}}(y) d y .
$$




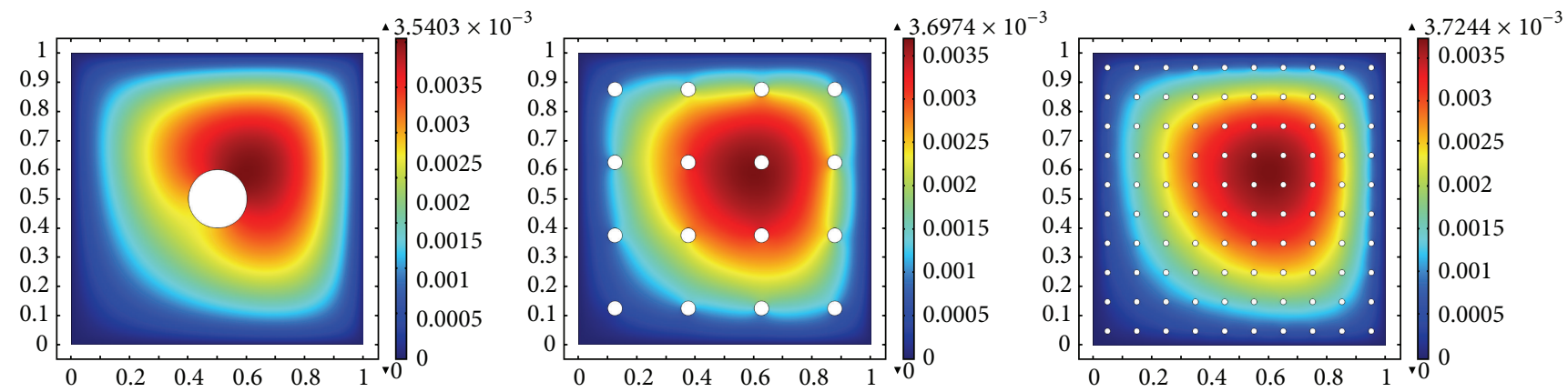

FIGURE 5: Level curves of solutions in the example, with $\varepsilon=0.1,0.025$, and 0.01 .

Thus, we can obtain $\mathbb{E}\left(X_{t}\right)$, which now can be used for solving the inverse problem for (45) via the method illustrated in Section 2. We recover the function

$$
\Phi_{t}=\int_{D} g(\tilde{u}(t, y)) f_{X_{t}}(y) d y .
$$

The last step involves the analysis of (47). The only unknown in this model is $g$. Taking the $L^{2}$ expansions of $\Phi$ and $g$ with respect to the same $L^{2}$ orthonormal basis $\left\{\phi_{i}\right\}$, we get

$$
\sum_{i \geq 0} b_{i} \phi_{i}=\sum_{i \geq 0} a_{i} \int_{D} \phi_{i}(\tilde{u}(t, y)) f_{X_{t}}(y) d y,
$$

and then

$$
b_{j}=\sum_{i \geq 0} a_{i} \int_{\mathbb{R}}\left[\int_{D} \phi(\tilde{u}(s, y)) f_{X_{s}}(y) d y\right] \phi_{j}(s) d s,
$$

for $j \geq 0$, a linear system in $a_{i}$, the solution of which is our final step.

4.2. Inverse Problems on Perforated Domains. A porous medium (or perforated domain) is a material characterized by a partitioning of the total volume into a solid portion often called the "matrix" and a pore space usually referred to as "holes." Mathematically speaking, these holes can be either materials different from those of the matrix or real physical holes. When formulating differential equations over porous media, the term "porous" implies that the state equation is written in the matrix only while boundary conditions should be imposed on the whole boundary of the matrix, including the boundary of the holes. Examples of this are Stokes or Navier-Stokes equations that are usually written only in the fluid part while the rocks play the role of "mathematical" holes. Porous media are encountered everywhere in real life and the concept of porous media is essential in many areas of applied sciences and engineering including petroleum engineering, chemical engineering, civil engineering, aerospace engineering, soil science, geology, and material science.

Since porosity in materials can take different forms and appear in varying degrees, solving differential equations over porous media is often a complicated task. Indeed, the size of holes and their distribution within a material play an important role in its characterization, and simulations conducted over porous media that include a large number of matrix-holes interfaces present real challenges. This is due to the need for a very fine discretization mesh which often requires a significant computational time and might even sometimes be irrelevant. This major difficulty is usually overcome by using the mathematical theory of "homogenization," where the heterogeneous material is replaced by a fictitious homogeneous one through a delicate approach that is not simply an averaging procedure. Several techniques are currently in use in homogenization including the multiple scale method, the method of oscillating test functions of Tartar, the two-scale convergence method, and, most recently, the periodic unfolding method.

In the case of porous media, or heterogeneous media in general, characterizing the properties of the material is a tricky process and can be done on different levels, mainly the microscopic and macroscopic scales, where the microscopic scale describes the heterogeneities and the macroscopic one describes the global behavior of the composite. To provide a numerical example of an inverse problem on a perforated domain, we set $\Omega=[0,1]^{2}$ and for $0<\varepsilon \leq 0.1$ define $N_{\varepsilon}=1 / 10 \varepsilon$ and

$$
\Omega_{\varepsilon}=\bigcup_{i, j=1}^{N_{\varepsilon}} B_{\varepsilon}\left(\left(i-\frac{1}{2}\right) \varepsilon,\left(j-\frac{1}{2}\right) \varepsilon\right),
$$

a domain with $N_{\varepsilon}^{2}$ uniformly distributed holes of radius $\varepsilon$. Choosing $K(x, y)=K_{\text {true }}(x, y)=10+2 x+3 y$, we consider the steady-state diffusion problem

$$
\begin{aligned}
\nabla \cdot(K(x, y) \nabla u(x, y)) & =x^{2}+y^{2}, \quad(x, y) \in \Omega \backslash \Omega_{\varepsilon}, \\
u(x, y) & =0, \quad \partial \Omega, \\
\frac{\partial u}{\partial n}(x, y) & =0, \quad \partial \Omega_{\varepsilon} .
\end{aligned}
$$

Suppose that, for a certain $\epsilon$, we sample the solution $u_{\epsilon}^{\lambda}$ of the above model on the perforated domain $\Omega_{\varepsilon}$. We aim at estimating $\lambda$ starting from this data and by using the model which is obtained when $\epsilon=0$ instead. In fact, it is much easier to solve an inverse problem on the initial domain $\Omega$ instead of on the perforated domain $\Omega_{\epsilon}$.

For a fixed value of $\varepsilon$, we solve the diffusion problem numerically and sample the solution at $M \times M$ uniformly 
TABLE 5: Results for the inverse problem. True values are $\left(\lambda_{0}, \lambda_{1}, \lambda_{2}\right)=(10,2,3)$.

\begin{tabular}{|c|c|c|c|c|c|}
\hline \multirow{2}{*}{$\varepsilon$} & \multirow{2}{*}{$N_{\varepsilon}$} & \multirow{2}{*}{$M$} & \multicolumn{3}{|c|}{ Recovered parameters } \\
\hline & & & $\lambda_{0}$ & $\lambda_{1}$ & $\lambda_{2}$ \\
\hline \multirow{3}{*}{0.1} & \multirow{3}{*}{1} & 9 & 13.2068 & -0.5921 & 0.6250 \\
\hline & & 49 & 13.2428 & -0.5837 & 0.6346 \\
\hline & & 49 & 13.2419 & -0.5798 & 0.6398 \\
\hline \multirow{3}{*}{0.025} & \multirow{3}{*}{4} & 9 & 9.8434 & 1.8148 & 2.8119 \\
\hline & & 49 & 9.9758 & 1.6894 & 2.6875 \\
\hline & & 99 & 9.9787 & 1.6838 & 2.6820 \\
\hline \multirow{3}{*}{0.01} & \multirow{3}{*}{10} & 9 & 9.9811 & 1.6221 & 2.6199 \\
\hline & & 49 & 10.0069 & 1.6041 & 2.6014 \\
\hline & & 99 & 10.0069 & 1.6039 & 2.6014 \\
\hline
\end{tabular}
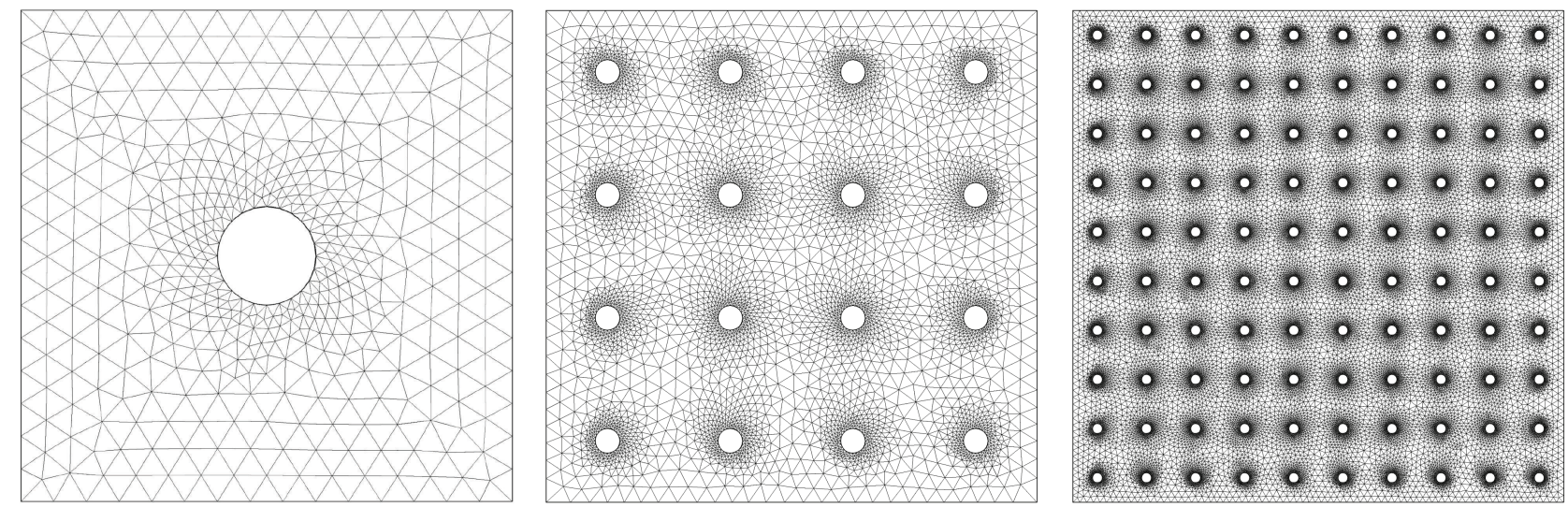

Figure 6: Meshes for each problem.

distributed points strictly inside $\Omega$. If such a point lies inside a hole, we obtain no information at the point. Using the $M^{2}$ (or fewer) data points, we use the Generalized Collage Theorem to solve the related inverse problem, seeking a diffusivity function of the form $K(x, y)=\lambda_{0}+\lambda_{1} x+\lambda_{2} y$.

For this example, we use the particular values $\varepsilon=0.1$, 0.025 , and 0.01 , corresponding to $N_{\varepsilon}=1,4$, and 10 . The level curves are illustrated in Figure 5. The results for $M=9,49$, and 99 are given in Table 5. In Figure 6, we illustrate the meshes used for numerically solving each of the problems.

\section{Conflict of Interests}

The authors declare that there is no conflict of interests regarding the publication of this paper.

\section{Acknowledgment}

H. Kunze and F. Mendivil were partially supported by NSERC in the form of a discovery grant.

\section{References}

[1] J. B. Keller, "Inverse problems," The American Mathematical Monthly, vol. 83, no. 2, pp. 107-118, 1976.
[2] A. Kirsch, An Introduction to the Mathematical Theory of Inverse Problems, vol. 120 of Applied Mathematical Sciences, Springer, New York, NY, USA, 2011.

[3] H. Kunze, D. la Torre, F. Mendivil, and E. R. Vrscay, FractalBased Methods in Analysis, Springer, New York, NY, USA, 2012.

[4] F. D. Moura Neto and A. J. da Silva Neto, An Introduction to Inverse Problems with Applications, Springer, New York, NY, USA, 2013.

[5] A. Tarantola, Inverse Problem Theory and Methods for Model Parameter Estimation, SIAM, Philadelphia, Pa, USA, 2005.

[6] C. R. Vogel, Computational Methods for Inverse Problems, SIAM, New York, NY, USA, 2002.

[7] J. Hadamard, Lectures on the Cauchy Problem in Linear Partial Differential Equations, Yale University Press, 1923.

[8] H. W. Engl and W. Grever, "Using the L-curve for determining optimal regularization parameters," Numerische Mathematik, vol. 69, no. 1, pp. 25-31, 1994.

[9] Y. L. Keung and J. Zou, "An efficient linear solver for nonlinear parameter identification problems," SIAM Journal on Scientific Computing, vol. 22, no. 5, pp. 1511-1526, 2000.

[10] J. Li and J. Zou, "A multilevel correction method for parameter identification," Inverse Problems, vol. 23, pp. 1759-1786, 2007.

[11] J. Milstein, “The inverse problem: estimation of kinetic parameters," in Modeling of Chemical Reaction Systems, K. Ebert et al., Ed., Springer, Berlin, Germany, 1981. 
[12] A. N. Tychonoff and V. Y. Arsenin, Solution of Ill-Posed Problems, Winston \& Sons, Washington, DC, USA, 1977.

[13] A. N. Tihonov, "On the solution of ill-posed problems and the method of regularization," Doklady Akademii Nauk SSSR, vol. 151, pp. 501-504, 1963.

[14] M. Barnsley, Fractals Everywhere, Academic Press, New York, NY. USA, 1989.

[15] M. F. Barnsley, V. Ervin, D. Hardin, and J. Lancaster, "Solution of an inverse problem for fractals and other sets," Proceedings of the National Academy of Sciences of the United States of America, vol. 83, no. 7, pp. 1975-1977, 1986.

[16] B. Forte and E. R. Vrscay, "Inverse problem methods for generalized fractal transforms," in Fractal Image Encoding and Analysis, Y. Fisher, Ed., vol. 159 of NATO ASI Series F, Springer, New York, NY, USA, 1998.

[17] H. E. Kunze, J. E. Hicken, and E. R. Vrscay, "Inverse problems for ODEs using contraction maps and suboptimality of the 'collage method"' Inverse Problems, vol. 20, no. 3, pp. 977-991, 2004.

[18] H. E. Kunze and E. R. Vrscay, "Solving inverse problems for ordinary differential equations using the Picard contraction mapping," Inverse Problems, vol. 15, no. 3, pp. 745-770, 1999.

[19] H. Kunze, D. la Torre, and E. R. Vrscay, "Solving inverse problems for DEs using the collage theorem and entropy maximization," Applied Mathematics Letters, vol. 25, no. 12, pp. 23062311, 2012.

[20] H. Kunze and S. Gomes, "Solving an inverse problem for Urison-type integral equations using Banach's fixed point theorem," Inverse Problems, vol. 19, no. 2, pp. 411-418, 2003.

[21] H. E. Kunze, D. la Torre, and E. R. Vrscay, "Random fixed point equations and inverse problems using collage method for contraction mappings," Journal of Mathematical Analysis and Applications, vol. 334, no. 2, pp. 1116-1129, 2007.

[22] H. E. Kunze, D. La Torre, and E. R. Vrscay, "Inverse problems for random differential equations using the collage method for random contraction mappings," Journal of Computational and Applied Mathematics, vol. 223, no. 2, pp. 853-861, 2009.

[23] H. Kunze and D. Crabtree, "Using collage coding to solve inverse problems in partial differential equations," in Proceedings of the Maplesoft Conference, 2005.

[24] H. Kunze, D. La Torre, and E. R. Vrscay, "A generalized collage method based upon the Lax-Milgram functional for solving boundary value inverse problems," Nonlinear Analysis: Theory, Methods \& Applications, vol. 71, no. 12, pp. e1337-e1343, 2009.

[25] H. E. Kunze, D. La Torre, and E. R. Vrscay, "Solving inverse problems for variational equations using "generalized collage methods," with applications to boundary value problems," Nonlinear Analysis: Real World Applications, vol. 11, no. 5, pp. 37343743, 2010.

[26] K. M. Levere, H. Kunze, and D. La Torre, "A collage-based approach to solving inverse problems for second-order nonlinear parabolic PDEs," Journal of Mathematical Analysis and Applications, vol. 406, no. 1, pp. 120-133, 2013.

[27] V. Capasso, H. Kunze, D. La Torre, and E. R. Vrscay, "Parameter identification for deterministic and stochastic differentialequations," in Advances in Nonlinear Analysis: Theory, Methods and Applications, S. Sivasundaram et al., Ed., pp. 71-84, Cambridge Scientific, Cambridge, UK, 2009.

[28] V. Capasso, H. E. Kunze, D. la Torre, and E. R. Vrscay, "Solving inverse problems for biological models using the collage method for differential equations," Journal of Mathematical Biology, vol. 67, no. 1, pp. 25-38, 2013.
[29] V. Capasso, H. E. Kunze, D. La Torre, and E. R. Vrscay, “Solving inverse problems for differential equations by a "generalized collage" method and application to a mean field stochastic model," Nonlinear Analysis: Real World Applications, vol. 15, pp. 276-289, 2014.

[30] D. La Torre and S. Marsiglio, "Endogenous technological progress in a multi-sector growth model," Economic Modelling, vol. 27, no. 5, pp. 1017-1028, 2010.

[31] M. I. Berenguer, H. E. Kunze, D. La Torre, and M. R. Galán, "Galerkin schemes and inverse boundary value problems in reflexive Banach spaces," Journal of Computational and Applied Mathematics, vol. 275, pp. 100-112, 2015.

[32] M. I. Berenguer, H. E. Kunze, D. La Torre, and M. Ruiz Galán, "A collage-based approach to inverse problems for constrained variational equations," preprint.

[33] M. Ruiz Galán, "Characterization of the solvability of generalized constrained variational equations," Abstract and Applied Analysis, vol. 2012, Article ID 247425, 36 pages, 2012.

[34] E. M. Cabaña, "The vibrating string forced by white noise," Zeitschrift für Wahrscheinlichkeitstheorie und Verwandte Gebiete, vol. 15, pp. 111-130, 1970.

[35] E. Orsingher, "Randomly forced vibrations of a string," Annales de l'Institut Henri Poincaré B.: Calcul des Probabilités et Statistique, vol. 18, no. 4, pp. 367-394, 1982.

[36] V. Capasso, H. W. Engl, and S. Kindermann, "Parameter identification in a random environment exemplified by a multiscale model for crystal growth," Multiscale Modeling \& Simulation, vol. 7, no. 2, pp. 814-841, 2008. 


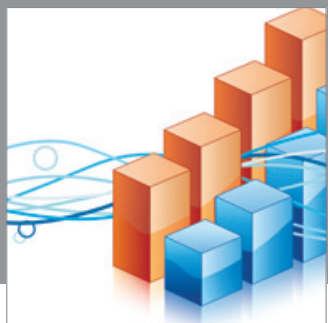

Advances in

Operations Research

mansans

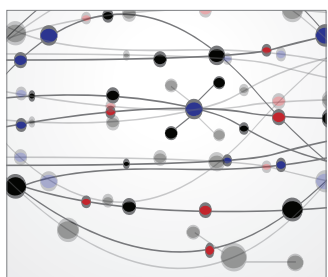

The Scientific World Journal
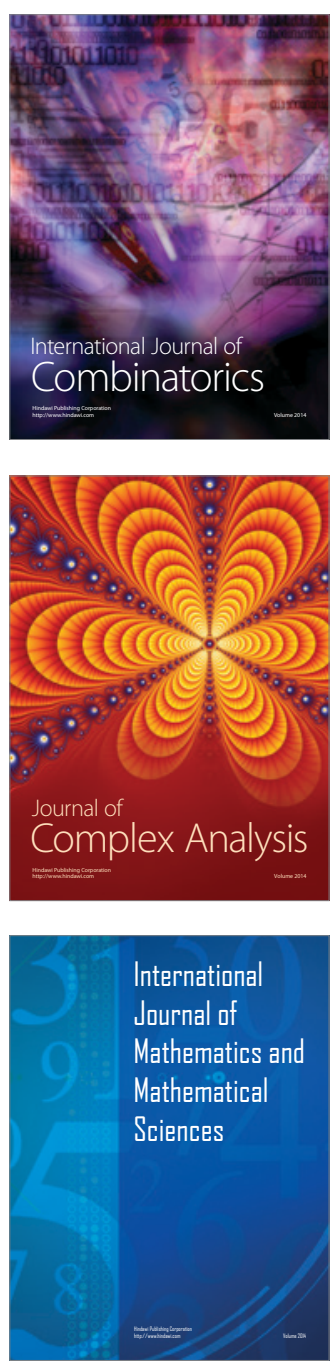
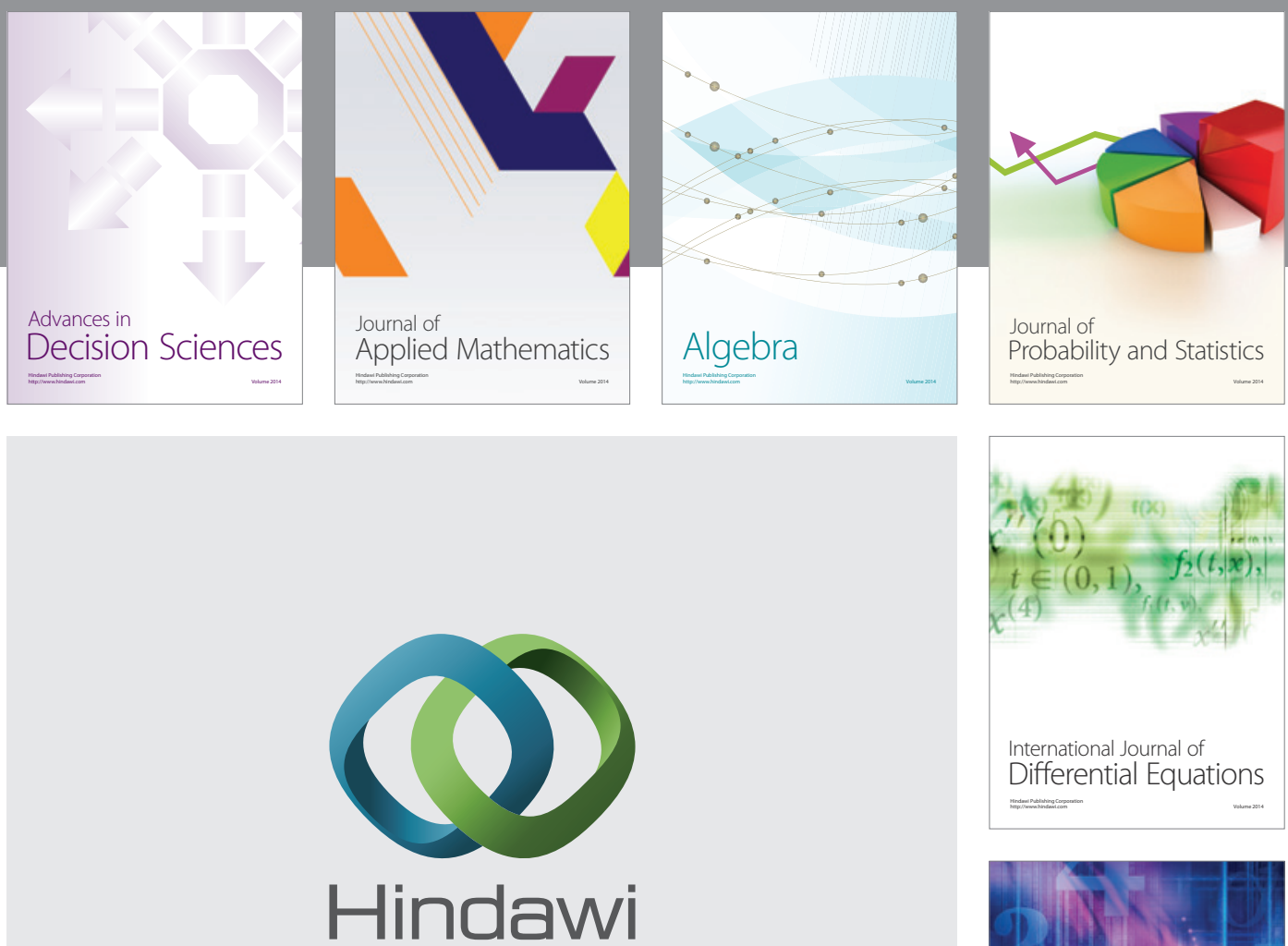

Submit your manuscripts at http://www.hindawi.com
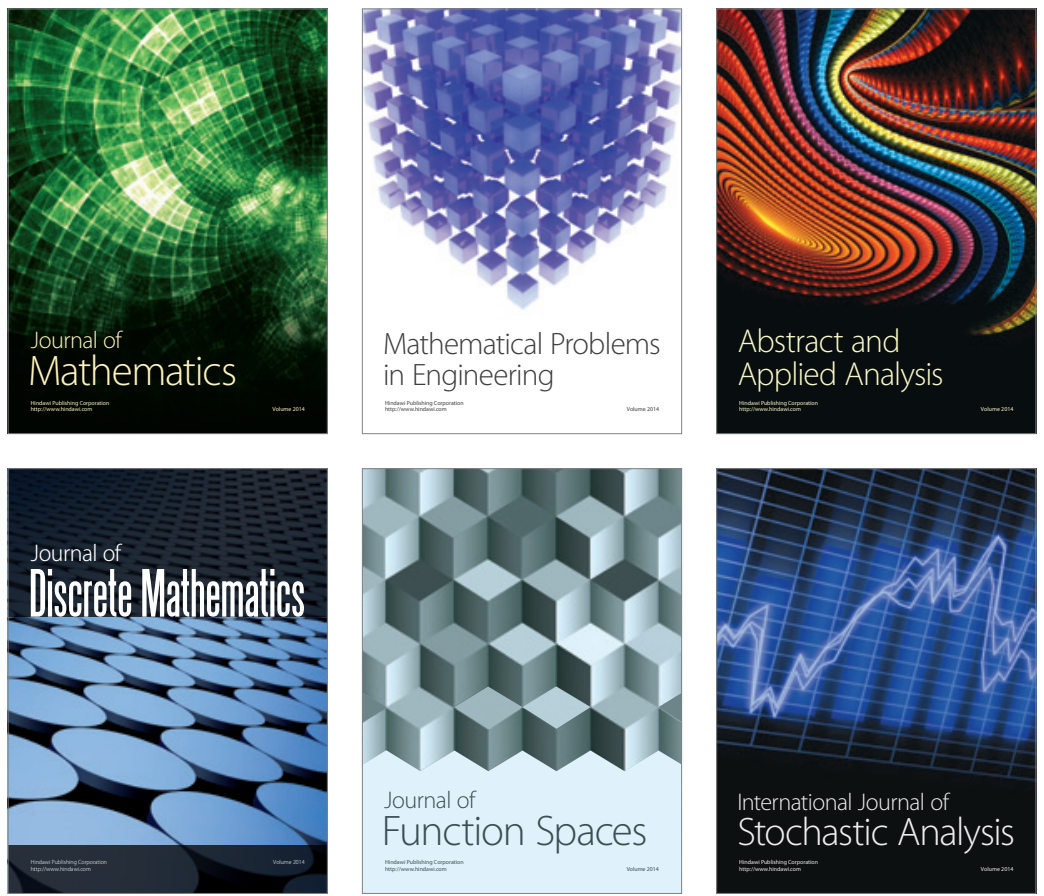

Journal of

Function Spaces

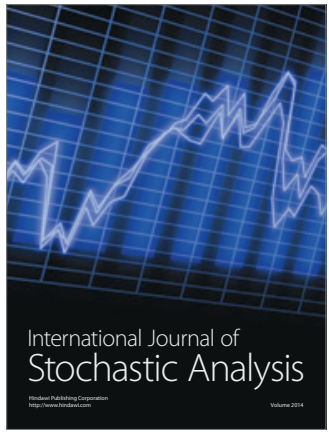

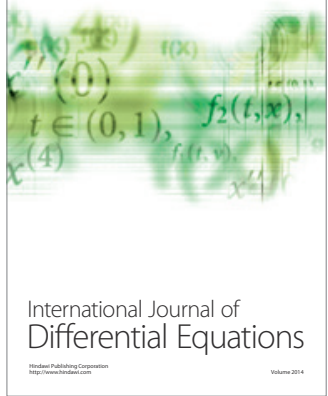
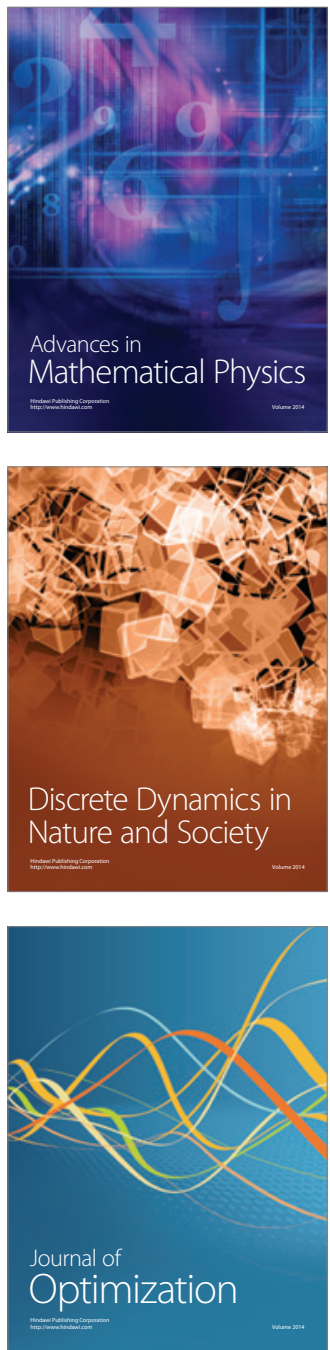